\title{
Measurement of Respiratory Chain Enzyme Activity in Human Renal Biopsy Specimens
}

\author{
Arun Ghose ${ }^{1}$, Christopher M. Taylor ${ }^{1}$, Alexander J. Howie ${ }^{2}$, Anapurna Chalasani ${ }^{3}$, \\ Iain Hargreaves ${ }^{3}$ and David V. Milford ${ }^{1, *}$ \\ 1 Department of Nephrology, Birmingham Children's Hospital, Birmingham B4 6NH, UK; \\ arun.ghose@bch.nhs.uk (A.G.); cmarktaylor@hotmail.com (C.M.T.) \\ 2 Department of Histopathology, Birmingham Children's Hospital, Birmingham B4 6NH, UK; \\ alexander.howie@nhs.net \\ 3 Neurometabolic Unit, National Hospital for Neurology and Neurosurgery, London WC1N 3BG, UK; \\ annapurnachalasani@nhs.net (A.C.); i.hargreaves@ucl.ac.uk (I.H.) \\ * Correspondence: david.milford@bch.nhs.uk; Tel.: +44-121-333-9228
}

Academic Editor: Mark S. Sands

Received: 6 July 2017; Accepted: 13 September 2017; Published: 19 September 2017

\begin{abstract}
Background: Mitochondrial disorders can present as kidney disease in children and be difficult to diagnose. Measurement of mitochondrial function in kidney tissue may help diagnosis. This study was to assess the feasibility of obtaining renal samples and analysing them for respiratory chain enzyme activity. Methods: The subjects were children undergoing a routine diagnostic renal biopsy, in whom a clinical condition of renal inflammation, scarring and primary metabolic disorder was unlikely. A fresh sample of kidney was snap frozen and later assayed for the activities of respiratory chain enzyme complexes I, II/III, and IV using spectrophotometric enzyme assay, and expressed as a ratio of citrate synthase activity. Results: The range of respiratory chain enzyme activity for complex I was 0.161 to 0.866 (mean 0.404 , SD 0.2 ), for complex II/III was 0.021 to 0.318 (mean 0.177, SD 0.095) and for complex IV was 0.001 to 0.025 (mean 0.015, SD 0.006). There were correlations between the different activities but not between them and the age of the children or a measure of the amount of chronic damage in the kidneys. Conclusion: It is feasible to measure respiratory chain enzyme activity in routine renal biopsy specimens.
\end{abstract}

Keywords: mitochondrial disorders; renal biopsy; respiratory chain enzymes

\section{Introduction}

Mitochondrial disorders are difficult to diagnose clinically because of phenotypic variation. Renal presentations include the renal Fanconi syndrome (tubular wasting of bicarbonate, sodium and potassium, glucose and amino acids) and steroid unresponsive nephrotic syndrome as a result of glomerular damage; progression to chronic kidney disease is also described [1-3]. Mitochondrial cytopathy is important to diagnose, as there may be reversibility of those with coenzyme Q10 biosynthesis defects as well as the implication for treatment options, including renal transplantation. A clue to the diagnosis may come from clinical abnormalities of other organs that require a high rate of oxidative metabolism, such as the brain (encephalopathy, retinopathy or stroke), skeletal muscle (myopathy), heart (cardiomyopathy) and liver; a full review of the clinical features of mitochondrial diseases is beyond the scope of this paper, but is extensively reviewed by Gorman et al. [4]. Without these other features, diagnosis may be difficult in children who have a predominantly renal presentation. Also, the proportion of normal to abnormal mitochondria may vary in different organs (heteroplasmy). Consequently, a diagnostic procedure performed on skeletal muscle, for example, may 
not identify an abnormality in a child whose main organ involvement is kidney or brain. A logical approach is to investigate tissue from the affected organ if possible [5-8].

Renal biopsy is a routine diagnostic procedure in children, principally to provide material for histopathological examination, but this can also be a source of appropriate tissue for enzymatic studies. Because mitochondrial disorders are likely to affect respiratory chain enzyme activity (RCEA), assay of this activity in renal biopsies could facilitate the diagnosis of these disorders. Before the measurement of RCEA could be investigated for its value in the diagnosis of mitochondrial disorders, it was necessary to see whether RCEA could be assayed in human renal tissue obtained by routine renal biopsy. The aim of this study was to determine the feasibility of measuring RCEA in renal biopsy specimens from children. The results of this study will provide pilot data for a more thorough validation study to assess the reliability of renal RCEA assessment as a diagnostic investigation.

\section{Experimental Section}

\subsection{Renal Biopsies}

Research and ethical approval was gained from the North Staffordshire Local Research Ethics Committee prior to the study (REC reference number: 08/H1204/123). Information for parents and information appropriate for the age of children was provided. Written parental consent was obtained, as was written consent from children over 14 years old.

Children aged from three to 16 years of age undergoing routine renal biopsy were invited to participate in the study. Samples were obtained using a 14-gauge biopsy needle to give two cores, and these were inspected immediately with a dissecting microscope. If there were more than $18 \mathrm{~mm}$ of cortex, a 3-mm piece of cortex was snap frozen in liquid nitrogen. In biopsies without $18 \mathrm{~mm}$ of cortex, and if there were more than $15 \mathrm{~mm}$ of medulla, a $3 \mathrm{~mm}$ core of medulla was taken. Only one research sample was taken from each patient. The remaining tissue was processed routinely for diagnosis. The frozen sample was only released for analysis of RCEA once the pathologist's report confirmed no more than minor structural abnormalities. If the diagnosis required more tissue, the frozen sample was formalin fixed for routine processing.

Biopsies from 13 children were considered suitable for RCEA assay. A sample from another child was not used as there was insufficient material for routine diagnostic studies. All these children had two kidneys of normal size, normal estimated glomerular filtration rate, and normal blood pressure. The indication for biopsy was persistent haematuria in six, nephrotic syndrome in four, persistent proteinuria in one, and treatment of the nephrotic syndrome with a calcineurin inhibitor, to check if chronic renal damage had resulted, in two. No child had any abnormalities outside the kidney to suggest a mitochondrial disorder.

Diagnoses were made in orthodox ways, and are given in the Results. Because some specimens had microscopically visible chronic damage, seen as tubular atrophy and/or global sclerosis of glomeruli, a morphometric method was used to measure this. Areas of chronic damage in the cortex were outlined on computer images of sections stained by periodic acid-methenamine silver, expressed as a percentage of the whole cortical cross-sectional area, and called the index of chronic damage [9].

\subsection{Tissue Homogenization}

Samples were immediately put on dry ice and then stored at $-70^{\circ} \mathrm{C}$. Before RCEA assay, samples were homogenized on ice, 1:9 (w/v), in $320 \mathrm{mmol} / \mathrm{L}$ sucrose, $1 \mathrm{mmol} / \mathrm{L}$ ethylenediamine tetra acetic acid dipotassium salt, and $10 \mathrm{mmol} / \mathrm{L}$ Trizma-base, $\mathrm{pH} 7.4$, using a pre-chilled hand-held glass homogenizer. Approximately 10-15 mg of renal tissue was homogenized per sample, distributed into four Eppendorf tubes. One Eppendorf tube was used for each enzyme determination following three cycles of freeze/thawing [7]. 


\subsection{RCEA Assays}

Mitochondrial respiratory chain enzyme and citrate synthase (CS) activities were determined by spectrophotometric enzyme assay [8,10]. Complex I (NADH:ubiquinone reductase, EC 1.6.5.3) activity was measured by the rotenone sensitive oxidation of NADH at $340 \mathrm{~nm}$. Complex II/III (succinate:cytochrome c reductase, EC 1.3.5.1 and EC 1.10.2.2) activity was measured by antimycin A-sensitive succinate dependent reduction of cytochrome c at $550 \mathrm{~nm}$. Complex IV (cytochrome c oxidase, EC 1.9.3.1) activity was measured by the potassium cyanide sensitive oxidation of reduced cytochrome $\mathrm{c}$ at $550 \mathrm{~nm}$. CS (EC 2.3.3.1) activity was determined by the formation of 5-thio-2-nitrobenzoic acid following the incubation of tissue homogenate with acetyl-CoA, oxaloacetate and 5,5'-dithiobis-(2-nitrobenzoic acid), at $412 \mathrm{~nm}$. No reference wavelength was used in the enzyme assays.

All mitochondrial RCEAs were expressed as a ratio to the activity of CS, a mitochondrial marker enzyme, to standardise the mitochondrial enrichment of the sample [11]. Because activities of CS and complexes I and II/III were expressed as $\mathrm{nmol} / \mathrm{min} / \mathrm{mL}$, but activity of complex IV was expressed as $\mathrm{k} / \mathrm{min} / \mathrm{mL}$, the ratios of complex I and complex II/III activities to CS activity have no units, but the ratio of complex IV activity to CS activity has units of $\mathrm{k} / \mathrm{nmol}$. The range, mean and standard deviation were calculated for each RCEA. Correlations between age, index of chronic damage and each RCEA were determined by Spearman's rank correlation coefficient $(r)$, and significant correlations were considered to be those with the conventional $p$ value of under 0.05 .

\section{Results}

Patient details, diagnoses, indexes of chronic damage, measurements of RCEA, and whether measurements were on cortex or medulla or on undetermined renal tissue are given in Table 1.

The range of RCEA for renal complex I was 0.161 to 0.866 (mean 0.404, SD 0.2), for complexes II/III was 0.021 to 0.318 (mean 0.177, SD 0.095) and for complex IV was 0.001 to 0.025 (mean 0.015, SD 0.006). There were no significant correlations between age and index of chronic damage, age and any RCEA, or index of chronic damage and any RCEA. RCEA of complex I showed a trend to correlate with RCEA of complexes II/III $(r=0.539, p=0.057)$ and was significantly correlated with RCEA of complex IV ( $r=0.704, p=0.007)$. RCEA of complexes II/III was significantly correlated with RCEA of complex IV $(r=0.660, p=0.014)$. The inter-assay coefficient of variation values for the RCEA and CS enzyme measurements calculated from the in-house QC human skeletal muscle homogenate data are as follows: Complex I: 9.11\% $(n=29)$; Complex II/III: 10.70\% $(n=29)$; Complex IV: $8.9 \%(n=29)$; CS: $5.6 \%(n=29)$. QC muscle samples were prepared from skeletal muscle tissue from patients with no biochemical evidence of a RCEA defect by the same method as the kidney homogenates were prepared. 
Table 1. Clinical details, index of chronic damage, and assay of respiratory chain enzyme activities (RCEA) in cortex or medulla, if site of tissue known. RCEA are expressed as a ratio of citrate synthase activity. Normal RCEA for skeletal muscle and liver are included for comparison [8].

\begin{tabular}{|c|c|c|c|c|c|c|}
\hline Sex, Age (year) & Diagnosis & Index of Chronic Damage & Complex I & Complex II/III & $\begin{array}{l}\text { Complex IV } \\
\text { (k/nmol) }\end{array}$ & $\begin{array}{l}\text { Cortexor Medulla or } \\
\text { Not Known }\end{array}$ \\
\hline M3 & minimal change & $0 \%$ & 0.176 & 0.021 & 0.003 & Cortex \\
\hline M7 & minimal change & $3 \%$ & 0.161 & 0.166 & 0.015 & Cortex \\
\hline M9 & CNI effects & $4 \%$ & 0.542 & 0.042 & 0.018 & Cortex \\
\hline M10 & Alport syndrome & $2 \%$ & 0.522 & 0.253 & 0.025 & Cortex \\
\hline F11 & IgA nephropathy & $3 \%$ & 0.220 & 0.120 & 0.016 & Medulla \\
\hline F13 & IgA nephropathy & $3 \%$ & 0.229 & 0.105 & 0.001 & cortex \\
\hline M13 & CNI effects & $15 \%$ & 0.388 & 0.238 & 0.017 & Cortex \\
\hline F14 & thin gbm disease & $3 \%$ & 0.866 & 0.313 & 0.018 & not known \\
\hline F14 & IgA nephropathy & $5 \%$ & 0.569 & 0.225 & 0.016 & not known \\
\hline M15 & thin gbm disease & $0 \%$ & 0.516 & 0.224 & 0.018 & Cortex \\
\hline F15 & seg. sclerosis & $2 \%$ & 0.333 & 0.105 & 0.014 & Medulla \\
\hline F16 & no abnormality & $0 \%$ & 0.452 & 0.318 & 0.018 & Cortex \\
\hline M16 & minimal change & $0 \%$ & 0.278 & 0.172 & 0.017 & Cortex \\
\hline \multicolumn{7}{|c|}{ normal RCEA values for skeletal muscle and liver } \\
\hline & skeletal muscle & & $0.104-0.268$ & $0.040-0.204$ & $0.014-0.034$ & \\
\hline & liver & & $0.054-0.22$ & $0.057-0.204$ & $\begin{array}{l}0.011-0.031 \\
\text { Reference ranges for } \\
\text { RCEA in liver and } \\
\text { muscle are not } \\
\text { thought to be age } \\
\text { dependent according } \\
\text { to the study of } \\
\text { Chretien et al. [12] }\end{array}$ & \\
\hline
\end{tabular}




\section{Discussion}

The DNA coding for mitochondrial respiratory chain enzymes NADH-Q oxidoreductase (complex I), succinate-Q reductase (complex II), Q-cytochrome c oxidoreductase (complex III) and complex IV (cytochrome c oxidase) is a mixture of nuclear genes and mitochondrial genes. Most disorders of RCEA in children are caused by mutations in nuclear genes, although mutations in mitochondrial DNA have also been found [13]. In some cases, genetic screening does not give a diagnosis, and so further investigations may be required. Another problem with mitochondrial respiratory chain disorders is that mutations may only be expressed in affected tissues. Consequently, assessment of the affected organ may be appropriate. Confirmation of abnormal RCEA may then be helpful in determining the most appropriate genetic tests. This study has indicated that it is possible to measure RCEA in kidney tissue obtained by the routine clinical procedure of renal biopsy.

Using CS activity as a reference controlled for variables such as the mitochondrial enrichment of the sample [11]. There was a wide range of RCEA for each of the complexes, with a 5-fold difference between the minimum value and maximum value for complex I, a 15-fold difference for complex II/III, and a 25-fold difference for complex IV (Table 1), although RCEA of the different complexes had significant or nearly significant correlations between each other. The mean RCEA of complex I was higher than the ranges for skeletal muscle and liver, the mean RCEA of complex II/II was towards the upper end of the ranges for skeletal muscle and liver, and the mean RCEA of complex IV was towards the lower end of the ranges for skeletal muscle and liver, although the RCEA in kidney were derived from a small sample. We cannot also exclude the possibility that poor sample handling of some of the renal biopsies may have contributed to loss of RCEA and therefore may account for the wide range of enzyme activities [14].

This study has a number of limitations. The number of patients sampled was small and most children had patchy chronic damage as assessed by the index of chronic damage measured in the histological specimen. However, as there was no way of knowing if the sample used for RCEA contained areas of chronic damage and the percentage of the biopsy specimen affected by chronic change was generally low (greater than $5 \%$ in only one child) it is not unreasonable to consider the values obtained as being representative of activity in normal renal tissue. Unfortunately, the small sample size precluded repeat analyses to confirm reproducibility, and so could explain the range of values obtained. Furthermore, no child with known mitochondrial disease presented during the time of this study to allow comparison with the values we obtained. Some workers have proposed validating the methodology by undertaking RCEA studies in nephrectomy samples, as these would provide a larger amount of renal tissue and allow reproducibility studies. However, we believe these samples would be unrepresentative; firstly, because the tissue would be exposed to significant ischaemia following clamping of the renal artery prior to the nephrectomy; and secondly, because the nephrectomy specimen would predominantly comprise diseased tissue. Importantly, given the heterogeneity of mitochondrial respiratory chain disorders, a reference range derived from true ethical controls may be required to accurately determine RCEA dysfunction in human renal tissue.

We have shown no relationship between the age of the children and RCEA in renal tissue when enzyme activities are expressed as a ratio to CS. Although this has previously been reported for RCEA in muscle and liver [12], this is the first time it has been reported for renal tissue. This is important since it indicates that age-specific reference intervals may not be necessary for renal RCEA if enzyme activities are expressed as a ratio to CS activity. The site of origin of the tissue analysed, whether cortex or medulla, also appeared unimportant. However, at present it cannot be excluded that there may be a difference in RCEA between these two regions of the kidney and future studies to confirm or refute this will be undertaken.

The lack of a relation between the index of chronic damage and RCEA is encouraging for future studies of RCEA, because the measured activities presumably reflect those in surviving tubules, suggesting that assays can still be applied to samples from children with a suspected mitochondrial disorder but with chronic kidney disease. 
We have shown that it is feasible to obtain samples from routine renal biopsies and freeze them for later analysis of RCEA. However, as there was insufficient tissue to duplicate analysis, the effect of poor sample handling, together with uncertainty about whether tissue from the renal medulla or cortex have different RCEA, this study is unable to establish a RCEA reference interval for renal tissue. It will require a larger and more rigorous validation study using ethically obtained control tissue, as well as renal biopsies from patients with confirmed mitochondrial respiratory chain dysfunction, to establish a reference range and to confirm the diagnostic value of this test. It remains to be seen if such a study is possible, given the difficulty in obtaining sufficient tissue from human renal biopsy.

Acknowledgments: The authors are grateful to Susan Cavanagh for her invaluable help in freezing, storing and retrieving the samples for this study.

Author Contributions: C.M.T. and D.V.M. conceived the study and obtained specimens; A.J.H. undertook the pathology review; A.C. and I.H. performed the RCEA; A.G. wrote the initial draft and all authors subsequently contributed to and approved the final version.

Conflicts of Interest: The authors declare no conflict of interest.

\section{References}

1. Niaudet, P.; Rotiq, A. Renal involvement in mitochondrial cytopathies. Pediatr. Nephrol. 1996, 10, 368-373. [CrossRef] [PubMed]

2. Munnich, A.; Rustin, P. Clinical spectrum and diagnosis of mitochondrial disorders. Am. J. Med. Genet. Part A 2001, 106, 4-17. [CrossRef] [PubMed]

3. Rotiq, A. Renal disease and mitochondrial genetics. J. Nephrol. 2003, 16, 286-292.

4. Gorman, G.S.; Chinnery, P.F.; DiMauro, S.; Hirano, M.; Koga, Y.; McFarland, R.; Suomalainen, A.; Thorburn, D.R.; Zeviani, M.; Turnbull, D.M. Mitochondrial diseases. Nat. Rev. Dis. Primers 2016, 2, 1-22. [CrossRef] [PubMed]

5. Kirby, D.M.; Thorburn, D.R.; Turnbull, D.M.; Taylor, R.W. Biochemical assays of the respiratory chain complex activity. Methods Cell Biol. 2007, 80, 93-119. [PubMed]

6. Janssen, A.J.; Smeitink, J.A.; van den Heuval, L.P. Some practical aspects of providing a diagnostic service for respiratory chain defects. Ann. Clin. Biochem. 2003, 40, 3-8. [CrossRef] [PubMed]

7. Thorburn, D.R. Practical problems in detecting abnormal mitochondrial function and genomes. Hum. Reprod. 2015, 2, 57-67. [CrossRef]

8. Heales, S.J.R.; Hargreaves, I.P.; Olpin, S.E. Diagnosis of mitochondrial electron transport chain defects in small muscle biopsies. J. Inherit. Metab. Dis. 1996, 19, 76.

9. Howie, A.J.; Ferreira, M.A.S.; Adu, D. Prognostic value of simple measurement of chronic damage in renal biopsy specimens. Nephrol. Dial. Transplant. 2001, 16, 1163-1169. [CrossRef] [PubMed]

10. Duberley, K.E.C.; Abramov, A.Y.; Chalasani, A.; Heales, S.J.; Rahman, S.; Hargreaves, I.P. Human neuronal coenzyme Q10 deficiency results in global loss of mitochondrial respiratory chain activity, increased mitochondrial oxidative stress and reversal of ATP synthase activity: Implications for pathogenesis and treatment. J. Inherit. Metab. Dis. 2013, 36, 63-73. [CrossRef] [PubMed]

11. Selak, M.A.; de Chadarevian, J.P.; Melvin, J.J.; Grover, W.D.; Salganicoff, L.; Kaye, E.M. Mitochondrial activity in Pompe's disease. Pediatr. Nephrol. 2000, 23, 54-57. [CrossRef]

12. Chretien, D.; Rustin, P.; Bourgeron, T.; Rötig, A.; Saudubray, J.M.; Munnich, A. Reference charts for respiratory chain activities in human tissue. Clin. Chim. Acta 1994, 228, 53-70. [CrossRef]

13. Scaglia, F. Nuclear gene defects in mitochondrial disorders. Biochem. Mol. Anal. 2012, 837, 17-34.

14. Berger, A.; Bruschek, M.; Grethen, C.; Sperl, W.; Kofler, B. Poor storage and handling of tissue mimics mitochondrial DNA depletion. Diagn. Mol. Pathol. 2001, 10, 55-59. [CrossRef] [PubMed]

(C) 2017 by the authors. Licensee MDPI, Basel, Switzerland. This article is an open access article distributed under the terms and conditions of the Creative Commons Attribution (CC BY) license (http:/ / creativecommons.org/licenses/by/4.0/). 